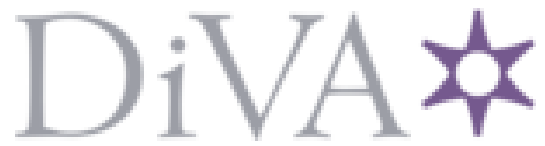

http://www.diva-portal.org

Preprint

This is the submitted version of a paper presented at 54th Annual Allerton Conference on Communication, Control, and Computing, Allerton 2016, Allerton HouseMonticello, United States, 27 September 2016 through 30 September 2016.

Citation for the original published paper:

Vu, M T., Oechtering, T J., Wiese, M., Skoglund, M. (2017)

Successive refinement with cribbing and side information

In: 54th Annual Allerton Conference on Communication, Control, and Computing, Allerton 2016, 7852212 (pp. 70-77). IEEE

https://doi.org/10.1109/ALLERTON.2016.7852212

N.B. When citing this work, cite the original published paper.

The work was supported by the Swedish Research Council (VR) project under Grant 2016-03853.

Permanent link to this version:

http://urn.kb.se/resolve?urn=urn:nbn:se:kth:diva-271119 


\title{
Successive refinement with cribbing and side information
}

\author{
Minh Thanh Vu, Tobias J. Oechtering, Moritz Wiese and Mikael Skoglund \\ School of Electrical Engineering and ACCESS Linnaeus Center \\ KTH Royal Institute of Technology, Stockholm, Sweden
}

\begin{abstract}
In this work we consider the successive refinement with side information where decoders cooperate via a cribbing link. Rate-distortion regions are characterized for non-causal side information and causal side information under appropriate constraints. An example is provided to illustrate the ratedistortion region for non-causal side information.
\end{abstract}

\section{INTRODUCTION}

The emerging big data paradigm motivates studies of efficient compression methods to store data in distributed storage systems subject to application constraints, e.g. delay constraints at end users' side. Moreover, advanced technologies and resource management enable cooperation between applications (users). Therefore, a progressive step is to study cooperative source coding problems.

Cooperation was initiated in the context of channel coding by Willems [1], [2] for multiple access channels (MACs), who introduced the concepts of conferencing and cribbing, respectively. In the conferencing scheme two encoders communicate over rate limited links, whereas in the cribbing approach each encoder obtains the output of the other encoder subject to causality constraints. Recently, the benefits of cooperation have been also studied under the presence of causal state information for MACs in [3] and non-causal state information for degraded broadcast channels in [4].

Cooperation has also been investigated in source coding in the form of a rate limited link between decoders which was initiated by the work of Yamamoto [5] who considered a cascade setup. In [6] Chia et al. considered cascade and triangular settings taking degraded side information into account. In [7] the notion of cribbing was transferred to the successive refinement scheme where the weaker decoder cribs the information from the output of the stronger one also subject to causality constraints. Therein cribbed information is viewed as a form of action dependent side information.

In this work we partially characterize the influence of side information to the cribbing scheme by putting them into the successive refinement setting. Specifically, we consider two scenarios. In the first one, we assume that degraded side information is present at decoders non-causally. Then, the cribbing is carried out non-causally. Our setting can be motivated from applications in which information is processed in block manner where the time constraint is not critical, for instance in data analytic applications. In some other applications subject to delay constraints [8] such as video streaming, it is required that Decoder 1 and Decoder 2 should provide the output simultaneously. In this scenario, the side information is causally and identically available at both decoders and non-causally available at the encoder. Additionally, Decoder 2 can crib the output of Decoder 1 strictly causally or causally. Other potential applications of these settings might be found in distributed sensor networks. Our results illuminate to some extent the dual roles of state information in channel coding and side information in source coding when cooperation is possible.

In the next section we state problem specifications and our main results. In Section III, the proofs of the results in Section II are provided. A numerical example that illustrates the non-causal achievable rate region is given in Section IV.

\section{Problem statement and Results}

We begin by introducing some notations. Random variables, their realizations and alphabets are denoted by uppercase, lower cases, and calligraphic letters, respectively. We consider only the finite alphabets where the source and side information $\left(X^{n}, Y^{n}, Z^{n}\right)$ are generated i.i.d. from the joint probability distribution $p_{X Y Z}$. The letter-wise distortion measures at both decoders are bounded mappings of the form $d: \mathcal{X} \times \hat{\mathcal{X}}_{k} \rightarrow\left[0, d_{\max }\right]$. With abuse of notation, the sequence distortion measures are defined as

$$
d\left(x^{n}, \hat{x}_{k}^{n}\right)=\frac{1}{n} \sum_{i=1}^{n} d\left(x_{i}, \hat{x}_{k i}\right)
$$

The random reconstruction symbols and their alphabets are denoted by $\hat{X}_{k}$ and $\hat{\mathcal{X}}_{k}$ for $k=1,2$. The strongly typical set is denoted by $\mathcal{T}_{\epsilon}^{n}$ and $[x]_{+}=\max \{x, 0\}$.

\section{A. Non-causal cribbing}

We first look at the scenario that side information $Y^{n}, Z^{n}$ is non-causally available at both decoders and the cribbing is perfect. The side information at the second decoder is a degraded version of the one at the first decoder, i.e., $X-Y-Z$. Moreover, $Y^{n}$ has the form $Y_{i}=h\left(X_{i}\right)$ for all $i=1, \ldots, n$, where $h(\cdot)$ is a deterministic function. This assumption subsumes the case that the source sequence is of the form $\left(\tilde{X}^{n}, \tilde{Y}^{n}\right)$ and the side information $\tilde{Y}^{n}$ is available at the first decoder. The side information $Y^{n}$ could be an independent quantization of the source obtained from other storage nodes or applications, i.e., our model can be viewed as a component of a bigger system. The scenario is illustrated in Fig. 1. We begin with definitions of a code and achievability.

Definition 1: A non-causal cribbing $\left(n, \mathcal{M}_{0}, \mathcal{M}_{1}\right)$-code of block length $n$ and message sets $\mathcal{M}_{0}, \mathcal{M}_{1}$ is defined via 


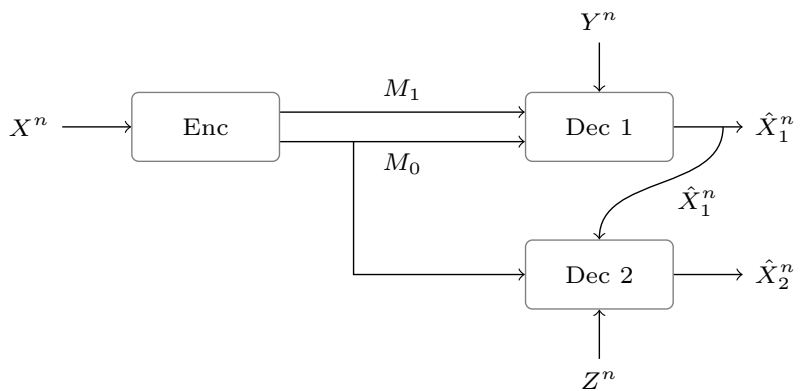

Fig. 1: Perfect cribbing of $\hat{X}_{1}^{n}$ where non-causal side information is available at both decoders. The side information satisfies $X-Y-Z$.

encoding functions $\phi_{k}: \mathcal{X}^{n} \rightarrow \mathcal{M}_{k}, k=0,1$, and decoding functions

$$
\begin{aligned}
& \psi_{1}: \mathcal{M}_{0} \times \mathcal{M}_{1} \times \mathcal{Y}^{n} \rightarrow \hat{\mathcal{X}}_{1}^{n}, \\
& \psi_{2}: \mathcal{M}_{0} \times \hat{\mathcal{X}}_{1}^{n} \times \mathcal{Z}^{n} \rightarrow \hat{\mathcal{X}}_{2}^{n} .
\end{aligned}
$$

Definition 2: A rate-distortion tuple $\left(R_{0}, R_{1}, D_{1}, D_{2}\right)$ is achievable if for every $\epsilon>0$ there exists an $\left(n, \mathcal{M}_{0}, \mathcal{M}_{1}\right)$ code such that

$$
\begin{aligned}
\frac{1}{n} \log \left|\mathcal{M}_{k}\right| & <R_{k}+\epsilon, k=0,1, \\
\mathbb{E}\left[d\left(X^{n}, \hat{X}_{k}^{n}\right)\right]<D_{k}+\epsilon, k & =1,2,
\end{aligned}
$$

for sufficiently large $n$. The set of all achievable ratedistortion tuples is the rate-distortion region and denoted by $\mathcal{R}_{1}$.

Next, we define an auxiliary single-letter rate-distortion region as follows.

Definition 3: Let $\mathcal{R}_{1}^{\star}$ be the set of rate-distortion tuples $\left(R_{0}, R_{1}, D_{1}, D_{2}\right)$ for which there exist auxiliary random variables $(U, V)$, taking values on finite sets $(\mathcal{U}, \mathcal{V})$, and deterministic reconstruction mappings

$$
\begin{aligned}
g_{1}: \mathcal{V} \times \mathcal{Y} & \rightarrow \hat{\mathcal{X}}_{1}, \\
g_{2}: \mathcal{U} \times \hat{\mathcal{X}}_{1} \times \mathcal{Z} & \rightarrow \hat{\mathcal{X}}_{2},
\end{aligned}
$$

with $\hat{X}_{1}=g_{1}(V, Y)$ and $\hat{X}_{2}=g_{2}\left(U, \hat{X}_{1}, Z\right)$ such that the following requirements are satisfied

$$
\begin{aligned}
& U-V-X-Y-Z, \\
& R_{0} \geq {\left[I\left(X ; U, \hat{X}_{1} \mid Z\right)-H\left(\hat{X}_{1} \mid Z\right)\right]_{+}, } \\
& R_{0}+R_{1} \geq {\left[I\left(X ; U, \hat{X}_{1} \mid Z\right)-H\left(\hat{X}_{1} \mid Z\right)\right]_{+} } \\
&+I\left(X ; V \mid U, \hat{X}_{1}, Y\right), \\
& R_{0}+R_{1} \geq I(X ; V \mid Y), \\
& D_{1} \geq \mathbb{E}\left[d\left(X, g_{1}(V, Y)\right)\right], \\
& D_{2} \geq \mathbb{E}\left[d\left(X, g_{2}\left(U, \hat{X}_{1}, Z\right)\right)\right] .
\end{aligned}
$$

Our first result can be summarized in the following theorem.

Theorem 1: The rate-distortion region $\mathcal{R}_{1}$ is given by

$$
\mathcal{R}_{1}=\mathcal{R}_{1}^{\star} .
$$

Next, we limit the capability of Decoder 2 by assuming that it can only crib a quantized version of Decoder 1's output, i.e., the second decoding function is given by

$$
\psi_{2}: \mathcal{M}_{0} \times \hat{\mathcal{T}}_{1}^{n} \times \mathcal{Z}^{n} \rightarrow \hat{\mathcal{X}}_{2}^{n},
$$

where $\hat{T}_{1 i}=q\left(\hat{X}_{1 i}\right)$ for all $i=1, \ldots, n$, and $q(\cdot)$ is a deterministic function. The following proposition characterizes the achievable rate-distortion region under the given restriction.

Proposition 1: The rate-distortion region, $\mathcal{R}_{1}^{\prime}$, is obtained from (5) by replacing $\hat{X}_{1}$ with $\hat{T}_{1}$ in the rate and distortion expressions.

Remark: Assuming that the side information $Z$ is nondegenerate, then setting $q(\cdot)=$ const in Proposition 1 gives us the successive refinement region in [9].

\section{B. Cribbing with identical causal side information}

For delay-limited applications, we study a special case for which we are able to fully characterize the rate-distortion region. The side information is available at both decoders identically causally and at encoder non-causally. In comparison to Section II-A, we make no assumption about the form of side information in this scenario. At first, the cribbing is restricted to be strictly causal, i.e., in producing the $i$-th output symbol Decoder 2 has access to Decoder 1 's reconstruction sequence $\hat{X}_{1}^{i-1}$ and the side information sequence $Y^{i}$. The set up is depicted in Fig. 2.

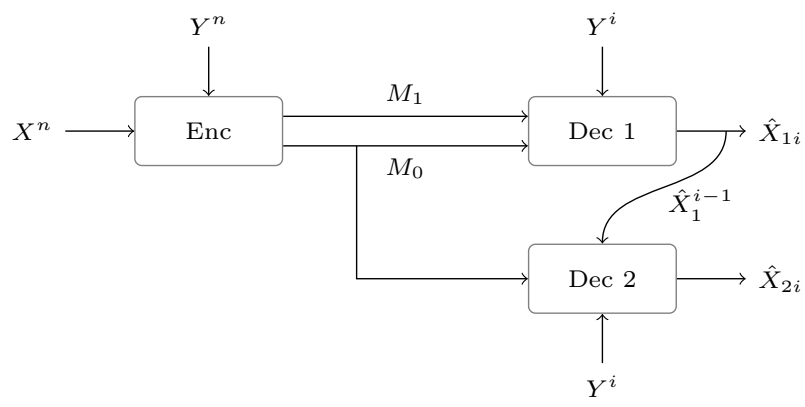

Fig. 2: Identically causal side information at both decoders with strictly causal cribbing, i.e., $\hat{X}_{1}^{i-1}$ is available to Decoder 2 .

Definition 4: A stricly causal cribbing $\left(n, \mathcal{M}_{0}, \mathcal{M}_{1}\right)$ code consists of encoding functions

$$
\phi_{k}: \mathcal{X}^{n} \times \mathcal{Y}^{n} \rightarrow \mathcal{M}_{k}, k=0,1,
$$

and two sets of decoding functions

$$
\begin{aligned}
\psi_{1 i}: \mathcal{M}_{0} \times \mathcal{M}_{1} \times \mathcal{Y}^{i} & \rightarrow \hat{\mathcal{X}}_{1}, \\
\psi_{2 i}: \mathcal{M}_{0} \times \hat{\mathcal{X}}_{1}^{i-1} \times \mathcal{Y}^{i} & \rightarrow \hat{\mathcal{X}}_{2}, i=1, \ldots, n .
\end{aligned}
$$

The definition of achievability is identical to the one in II-A. We denote the rate-distortion region by $\mathcal{R}_{2}$.

Theorem 2: The rate-distortion region $\mathcal{R}_{2}$ is characterized by

$$
R_{0} \geq\left[I\left(X ; U, \hat{X}_{1} \mid Y\right)-H\left(\hat{X}_{1} \mid U, Y\right)\right]_{+},
$$




$$
\begin{aligned}
& R_{0}+R_{1} \geq I\left(X ; U, \hat{X}_{1} \mid Y\right), \\
& D_{1} \geq \mathbb{E}\left[d\left(X, \hat{X}_{1}\right)\right], D_{2} \geq \mathbb{E}\left[d\left(X, g_{2}(U, Y)\right)\right],
\end{aligned}
$$

for some deterministic reconstruction mapping $g_{2}: \mathcal{U} \times \mathcal{Y} \rightarrow$ $\hat{\mathcal{X}}_{2}$ and conditional distribution $p_{U \hat{X}_{1} \mid X Y}$.

If the cribbing is also allowed causally, i.e., the second set of decoding functions becomes

$$
\psi_{2 i}: \mathcal{M}_{0} \times \hat{\mathcal{X}}_{1}^{i} \times \mathcal{Y}^{i} \rightarrow \hat{\mathcal{X}}_{2}, i=1, \ldots, n,
$$

then the rate-distortion region is described by the following proposition.

Proposition 2: The rate-distortion region, $\mathcal{R}_{2}^{\prime}$, can be obtained from (10) by replacing the distortion constraint at Decoder 2 by

$$
D_{2} \geq \mathbb{E}\left[d\left(X, g_{2}\left(U, \hat{X}_{1}, Y\right)\right)\right]
$$

\section{PROOFS}

In the process of proving Theorem 1, we first show a general result where $X, Y, Z$ are arbitrarily correlated.

Proposition 3: The following set of rate-distortion tuples $\left(R_{0}, R_{1}, D_{0}, D_{1}\right)$ is achievable

$$
\begin{aligned}
& R_{0} \geq[ {\left[(X ; U, V \mid Z)-I\left(U, V ; \hat{X}_{1} \mid Z\right)\right]_{+}, } \\
& R_{0}+R_{1} \geq[\left.I(X ; U, V \mid Z)-I\left(U, V ; \hat{X}_{1} \mid Z\right)\right]_{+} \\
&+I(X ; W \mid U, V, Y), \\
& R_{0}+R_{1} \geq I(X ; U, V, W \mid Y), \\
& D_{1} \geq \mathbb{E}\left[d\left(X, g_{1}(W, Y)\right)\right] \\
& D_{2} \geq \mathbb{E}\left[d\left(X, g_{2}\left(U, \hat{X}_{1}, Z\right)\right)\right]
\end{aligned}
$$

for some conditional p.m.f $p_{U V W \mid X}$ and deterministic reconstruction mappings $g_{1}: \mathcal{W} \times \mathcal{Y} \rightarrow \hat{\mathcal{X}}_{1}$ and $g_{2}: \mathcal{U} \times \hat{\mathcal{X}}_{1} \times \mathcal{Z} \rightarrow$ $\hat{\mathcal{X}}_{2}$.

Proof: Fix a conditional distribution $p_{U V W \mid X}$ and two deterministic reconstruction mappings $g_{1}$ and $g_{2}$ such that the distortion constraints in (13) are satisfied. The resulting p.m.f is given by $p_{X Y Z} p_{U V W \mid X} p_{\hat{X}_{1} \mid W Y} p_{\hat{X}_{2} \mid U \hat{X}_{1} Z}$, where the last two terms take values in the set $\{0,1\}$. In the following, we first provide an overview of our approach.

Our aim is to simplify the expression of the common rate $R_{0}$. Assume that the first two layers of information are described by $U$ and $V$, with the corresponding code rate $R_{U}$ and $R_{V}$, respectively. We employ non-unique decoding to recover $u^{n}$ with the help of $v^{n}$. More specifically, we look for a unique $u^{n}$, using its binning index, such that the tuple $\left(u^{n}, v^{n}, z^{n}, \hat{x}_{1}^{n}\right)$ is jointly typical for some sequence $v^{n}$. We want to fix the expression of the common rate as

$$
R_{0}=R_{U}+R_{V}-I\left(U, V ; Z, \hat{X}_{1}\right) .
$$

However, the ambiguous relation between $I(U, V ; Y)$ and $I\left(U, V ; Z, \hat{X}_{1}\right)$ poses a challenge, which then can be alleviated by using a nested binning approach. We illustrate different situations in Fig. 3. In case (a), we can use the extra advantage of Decoder 2 to reduce transmission rate on the common link to (14). In case (b), we can set the rates of $l_{u}, l_{v}$ to be negligible and still ensure that the common rate is given by (14).
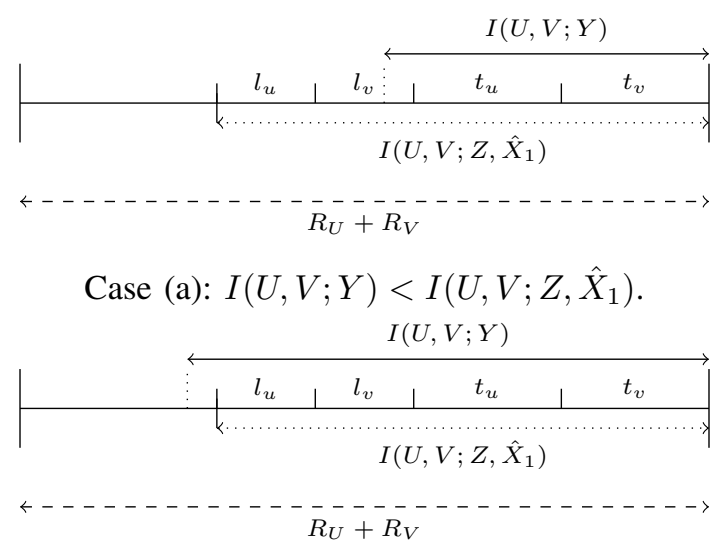

Case (b): $I(U, V ; Y) \geq I\left(U, V ; Z, \hat{X}_{1}\right)$.

Fig. 3: Illustration of nested binnings for two scenarios.

Codebook generation: We draw $2^{n R_{U}}$ sequences $u^{n}(m)$ i.i.d. according to the marginal distribution $p_{U}$. Each index $m$ is parsed into the triple $\left(m_{u}, l_{u}, t_{u}\right)$. We can view $m_{u}$ as a superbin index, $l_{u}$ as a subbin index, and so on.

For each sequence $u^{n}(m)$ we generate $2^{n R_{V}}$ i.i.d. sequences $v^{n}(m, l)$ according to the conditional distribution $p_{V \mid U}$. Similarly, we parse each index $l$ into a unique triple $\left(m_{v}, l_{v}, t_{v}\right)$.

Finally, for each pair of sequences $\left(u^{n}(m), v^{n}(m, l)\right)$ we generate $2^{n R_{W}}$ i.i.d. sequences $w^{n}(m, l, j)$ by the conditional distribution $p_{W \mid U V}$. Then, each index $j$ is parsed into a unique pair $\left(m_{w}, l_{w}\right)$. The codebook is revealed to the encoder and all decoders.

Encoding: Given a source sequence $x^{n}$, we look for a codeword $u^{n}(m)$ such that $\left(x^{n}, u^{n}(m)\right) \in \mathcal{T}_{\epsilon}^{n}$. Then, we look for a codeword $v^{n}(m, l)$ such that

$$
\left(x^{n}, u^{n}(m), v^{n}(m, l)\right) \in \mathcal{T}_{\epsilon}^{n} .
$$

Finally, we look for a codeword $w^{n}(m, l, j)$ such that

$$
\left(x^{n}, u^{n}(m), v^{n}(m, l), w^{n}(m, l, j)\right) \in \mathcal{T}_{\epsilon}^{n} .
$$

Note that in all of the above steps, if there is more than one suitable codewords, we select one of them uniformly at random. Otherwise, if no suitable codeword exists, a codeword is selected uniformly at random from the corresponding subcodebook. The corresponding pair of indices $\left(m_{u}, m_{v}\right)$ is sent as message $m_{0}$ on the common link to both decoders. In the private link to Decoder 1 we send the tuple of indices $\left(l_{u}, l_{v}, m_{w}\right)$ as message $m_{1}$.

Decoding: Decoder 1 searches for the unique index $\hat{t}_{u}$ such that $\left(u^{n}(\hat{m}), y^{n}\right) \in \mathcal{T}_{\epsilon}^{n}$, where $\hat{m}=\left(m_{u}, l_{u}, \hat{t}_{u}\right)$. Then, it looks for the unique index $\hat{t}_{v}$ such that $\left(u^{n}(\hat{m}), v^{n}(\hat{m}, \hat{l}), y^{n}\right) \in \mathcal{T}_{\epsilon}^{n}$, where $\hat{l}=\left(m_{v}, l_{v}, \hat{t}_{v}\right)$, and finally for the unique index $\hat{l}_{w}$ such that

$$
\left(u^{n}(\hat{m}), v^{n}(\hat{m}, \hat{l}), w^{n}(\hat{m}, \hat{l}, \hat{j}), y^{n}\right) \in \mathcal{T}_{\epsilon}^{n},
$$

where $\hat{j}=\left(m_{w}, \hat{l}_{w}\right)$. Then, an output sequence is produced as $\hat{x}_{1 i}=g_{1}\left(w_{i}(\hat{m}, \hat{l}, \hat{j}), y_{i}\right)$ for all $i=1, \ldots, n$. 
Decoder 2 looks for the unique pair of indices $\left(\tilde{l}_{u}, \tilde{t}_{u}\right)$ such that

$$
\left(u^{n}(\tilde{m}), v^{n}\left(\tilde{m}, l^{\prime}\right), z^{n}, \hat{x}_{1}^{n}\right) \in \mathcal{T}_{\epsilon}^{n},
$$

for some $l^{\prime}$, where $\tilde{m}=\left(m_{u}, \tilde{l}_{u}, \tilde{t}_{u}\right)$ and $l^{\prime}=\left(m_{v}, l_{v}^{\prime}, t_{v}^{\prime}\right)$. Then, it outputs the corresponding sequence $\hat{x}_{2}^{n}$ via $\hat{x}_{2 i}=$ $g_{2}\left(u_{i}(\tilde{m}), \hat{x}_{1 i}, z_{i}\right)$, for all $i=1, \ldots, n$.

Analysis: From the covering lemma [10, Lemma 3.3], the probability that the source sequence and the chosen codewords are jointly typical goes to one as $n \rightarrow \infty$ if

$$
\begin{aligned}
R_{m_{u}}+R_{l_{u}}+R_{t_{u}} & >I(X ; U)+\gamma_{n}, \\
R_{m_{v}}+R_{l_{v}}+R_{t_{v}} & >I(X ; V \mid U)+\gamma_{n}, \\
R_{m_{w}}+R_{l_{w}} & >I(X ; W \mid U, V)+\gamma_{n},
\end{aligned}
$$

where $\gamma_{n} \rightarrow 0$ as $n \rightarrow \infty$. From the conditional typicality lemma [10, p.27] (cf. [11, Lemma 15.8.1]) we obtain

$$
\left(Z^{n}, Y^{n}, X^{n}, U^{n}(\cdot), V^{n}(\cdot), W^{n}(\cdot)\right) \in \mathcal{T}_{\epsilon}^{n},
$$

with probability going to one as $n \rightarrow \infty$. Decoder 1 successfully identifies all the indices uniquely with probability going to one if (cf. [10, Lemma 11.1])

$$
\begin{aligned}
& R_{t_{u}}<I(U ; Y)-\gamma_{n}, \\
& R_{t_{v}}<I(V ; Y \mid U)-\gamma_{n} \\
& R_{l_{w}}<I(W ; Y \mid U, V)-\gamma_{n} .
\end{aligned}
$$

These inequalities further imply that the probability of the event

$$
\left(Z^{n}, Y^{n}, X^{n}, U^{n}(\cdot), V^{n}(\cdot), W^{n}(\cdot), \hat{X}_{1}^{n}\right) \in \mathcal{T}_{\epsilon}^{n},
$$

tends to one due to the conditional typicality lemma, and $\hat{X}_{1}=g_{1}(W, Y)$. Using the approach in [12, Lemma 1], it can be shown that Decoder 2 recovers the unique pair of indices with probability going to one if

$$
R_{l_{u}}+R_{t_{u}}+R_{l_{v}}+R_{t_{v}}<I\left(U, V ; Z, \hat{X}_{1}\right)-\gamma_{n} .
$$

Under these constraints, by the typical average lemma [10, p.26], the distortion levels at both decoders are bounded by

$$
\mathbb{E}\left[d\left(X^{n}, \hat{X}_{k}^{n}\right)\right]<D_{k}+\gamma_{n}, k=1,2 .
$$

Using the Fourier-Motzkin elimination method [10, Appendix D], [13] we obtain that the rate tuples

$$
\begin{aligned}
R_{0} & \geq\left[I(X ; U, V)-I\left(U, V ; Z, \hat{X}_{1}\right)\right]_{+}, \\
R_{1} & \geq I(X ; W \mid U, V, Y), \\
R_{0}+R_{1} & \geq I(X ; U, V, W \mid Y),
\end{aligned}
$$

are achievable. The rate splitting argument in [10], i.e., transferring some information on the private link to the common link, allows us to enlarge the rate region to (13).

\section{A. Proof of Theorem 1}

Achievability: Fix a conditional p.m.f such that $U-V-$ $X-Y-Z$ and two reconstruction mappings $g_{1}, g_{2}$ defined as in (4). Furthermore, let $\left(R_{0}, R_{1}, D_{1}, D_{2}\right)$ be a rate-distortion tuple satisfying the inequalities in (5). We notice that since $Y$ is a deterministic function of $X$ the Markov relation

$$
Z-Y-X-\left(\hat{X}_{1}, U, V\right)
$$

holds. Hence, we are allowed to take for Proposition 3 $U^{\prime}=U, V^{\prime}=\hat{X}_{1}$ and $W^{\prime}=V$ and define $g_{1}^{\prime}, g_{2}^{\prime}$ correspondingly as $g_{1}^{\prime}\left(W^{\prime}, Y\right)=g_{1}(V, Y)$ and $g_{2}^{\prime}\left(U^{\prime}, \hat{X}_{1}^{\prime}, Z\right)=$ $g_{2}\left(U, \hat{X}_{1}, Z\right)$. Furthermore, we observe that plugging the random variables $\left(U^{\prime}, V^{\prime}, W^{\prime}\right)$ and mappings $g_{1}^{\prime}, g_{2}^{\prime}$ into the right-hand sides of (13) gives us the right-hand sides of (5). This implies that the tuple $\left(R_{0}, R_{1}, D_{1}, D_{2}\right)$ is achievable by Proposition 3.

Converse: Assume that there exists a non-causal cribbing code of length $n$ such that the rate-distortion tuple $\left(R_{0}, R_{1}, D_{1}, D_{2}\right)$ is achievable. For simplicity we denote $M_{k}=\phi_{k}\left(X^{n}\right)$ for $k=0,1$ and $Y^{n \backslash i}=\left(Y_{1}, \ldots, Y_{i-1}\right.$, $\left.Y_{i+1}, \ldots, Y_{n}\right)$. Similar abbreviations apply also for $Z$ and $\hat{X}_{1}$. Furthermore, we define

$$
\begin{aligned}
U_{i} & =\left(M_{0}, Y^{i-1}, Z^{n \backslash i}, \hat{X}_{1}^{n \backslash i}\right), \\
V_{i} & =\left(U_{i}, M_{1}, Y_{i+1}^{n}, X^{i-1}\right), i=1, \ldots, n .
\end{aligned}
$$

Since $Y_{i}$ is a deterministic function of $X_{i}$, for all $i=$ $1, \ldots, n$, we obtain the following Markov relation

$$
Z_{i}-Y_{i}-X_{i}-V_{i}-U_{i}, \forall i=1, \ldots, n .
$$

Note that (24) does not hold in general even if $X_{i}-Y_{i}-Z_{i}$, since $\hat{X}_{1}^{n}$ is a function of $M_{0}, M_{1}, Y^{n}$. Using these notations, the rate constraints can be expressed as

$$
\begin{aligned}
& n\left(R_{0}+\epsilon\right) \geq H\left(M_{0} \mid Z^{n}\right)=I\left(X^{n} ; M_{0} \mid Z^{n}\right) \\
&= I\left(X^{n} ; M_{0}, \hat{X}_{1}^{n} \mid Z^{n}\right)-I\left(X^{n} ; \hat{X}_{1}^{n} \mid Z^{n}, M_{0}\right) \\
& \geq I\left(X^{n} ; M_{0}, \hat{X}_{1}^{n} \mid Z^{n}\right)-H\left(\hat{X}_{1}^{n} \mid Z^{n}\right) \\
&= \sum_{i=1}^{n} I\left(X_{i} ; M_{0}, X^{i-1}, Z^{n \backslash i}, \hat{X}_{1}^{n \backslash i}, \hat{X}_{1 i} \mid Z_{i}\right) \\
& \quad-H\left(\hat{X}_{1 i} \mid Z_{i}, Z^{n \backslash i}, \hat{X}_{1}^{i-1}\right) \\
& \geq \sum_{i=1}^{n} I\left(X_{i} ; M_{0}, X^{i-1}, Z^{n \backslash i}, \hat{X}_{1}^{n \backslash i}, \hat{X}_{1 i} \mid Z_{i}\right)-H\left(\hat{X}_{1 i} \mid Z_{i}\right) \\
& \stackrel{(a)}{=} \sum_{i=1}^{n} I\left(X_{i} ; M_{0}, X^{i-1}, Y^{i-1}, Z^{n \backslash i}, \hat{X}_{1}^{n \backslash i}, \hat{X}_{1 i} \mid Z_{i}\right) \\
& \quad-H\left(\hat{X}_{1 i} \mid Z_{i}\right) \\
& \geq \sum_{i=1}^{n} I\left(X_{i} ; U_{i}, \hat{X}_{1 i} \mid Z_{i}\right)-H\left(\hat{X}_{1 i} \mid Z_{i}\right) .
\end{aligned}
$$

Note that $(a)$ is valid since $Y_{i}$ is a deterministic function of $X_{i}$ for all $i=1, \ldots, n$. Similarly

$$
\begin{aligned}
& n\left(R_{0}+R_{1}+\epsilon\right) \geq H\left(M_{0}, M_{1} \mid Y^{n}\right)=H\left(M_{0}, M_{1}, \hat{X}_{1}^{n} \mid Y^{n}\right) \\
& =I\left(X^{n} ; M_{0}, M_{1}, \hat{X}_{1}^{n} \mid Y^{n}\right)
\end{aligned}
$$




$$
\begin{aligned}
& =\sum_{i=1}^{n} I\left(X_{i} ; M_{0}, M_{1}, Y^{n \backslash i}, X^{i-1}, \hat{X}_{1 i}, \hat{X}_{1}^{n \backslash i} \mid Y_{i}\right) \\
& \stackrel{(b)}{=} \sum_{i=1}^{n} I\left(X_{i} ; M_{0}, M_{1}, Y^{n \backslash i}, X^{i-1}, Z^{n \backslash i}, \hat{X}_{1 i}, \hat{X}_{1}^{n \backslash i} \mid Y_{i}\right) \\
& =\sum_{i=1}^{n} I\left(X_{i} ; U_{i}, V_{i}, \hat{X}_{1 i} \mid Y_{i}\right)=\sum_{i=1}^{n} I\left(X_{i} ; V_{i} \mid Y_{i}\right) \\
& \geq \sum_{i=1}^{n} I\left(X_{i} ; V_{i} \mid U_{i}, \hat{X}_{1 i}, Y_{i}\right)
\end{aligned}
$$

and

$$
\begin{aligned}
& n\left(R_{0}+R_{1}+\epsilon\right) \geq H\left(M_{0}, M_{1} \mid Z^{n}\right) \\
& =I\left(Y^{n} ; M_{0}, M_{1} \mid Z^{n}\right)+I\left(X^{n} ; M_{0}, M_{1} \mid Y^{n}\right) \\
& \geq I\left(Y^{n} ; M_{0}, M_{1}, \hat{X}_{1}^{n} \mid Z^{n}\right)-H\left(\hat{X}_{1}^{n} \mid Z^{n}\right) \\
& \quad+I\left(X^{n} ; M_{0}, M_{1}, \hat{X}_{1}^{n} \mid Y^{n}\right) \\
& \quad \stackrel{(b)}{\geq} \sum_{i=1}^{n} I\left(Y_{i} ; M_{0}, Z^{n \backslash i}, Y^{i-1}, \hat{X}_{1}^{n \backslash i}, \hat{X}_{1 i} \mid Z_{i}\right)-H\left(\hat{X}_{1 i} \mid Z_{i}\right) \\
& \quad+I\left(X_{i} ; M_{0}, M_{1}, Y^{n \backslash i}, \hat{X}_{1}^{n \backslash i}, X^{i-1}, Z^{n \backslash i}, \hat{X}_{1 i} \mid Y_{i}\right) \\
& =\sum_{i=1}^{n} I\left(Y_{i} ; U_{i}, \hat{X}_{1 i} \mid Z_{i}\right)-H\left(\hat{X}_{1 i} \mid Z_{i}\right) \\
& \quad+I\left(X_{i} ; U_{i}, V_{i}, \hat{X}_{1 i} \mid Y_{i}\right) \\
& \stackrel{(c)}{=} \sum_{i=1}^{n} I\left(X_{i}, Y_{i} ; U_{i}, \hat{X}_{1 i} \mid Z_{i}\right)-H\left(\hat{X}_{1 i} \mid Z_{i}\right) \\
& \quad+I\left(X_{i} ; V_{i} \mid U_{i}, \hat{X}_{1 i}, Y_{i}\right) \\
& \stackrel{(a)}{=} \sum_{i=1}^{n} I\left(X_{i} ; U_{i}, \hat{X}_{1 i} \mid Z_{i}\right)-H\left(\hat{X}_{1 i} \mid Z_{i}\right) \\
& \quad+I\left(X_{i} ; V_{i} \mid U_{i}, \hat{X}_{1 i}, Y_{i}\right),
\end{aligned}
$$

where we use the Markov relations $Z^{n \backslash i}-Y^{n \backslash i}-$ $\left(Y_{i}, M_{0}, M_{1}, X^{i}, \hat{X}_{1}^{n}\right)$ in $(b)$ and $Z_{i}-Y_{i}-\left(X_{i}, U_{i}, V_{i}, \hat{X}_{1 i}\right)$ in (c) for all $i=1, \ldots, n$. Let $Q$ be a uniform random variable on the set $\{1, \ldots, n\}$ and independent of everything else. Additionally, we define $U=\left(U_{Q}, Q\right), V=\left(V_{Q}, Q\right)$. Then (25), (26), and (27) can be reformulated as

$$
\begin{aligned}
& R_{0}+\epsilon \geq {\left[I\left(X_{Q} ; U, \hat{X}_{1 Q} \mid Z_{Q}\right)-H\left(\hat{X}_{1 Q} \mid Z_{Q}, Q\right)\right]_{+} } \\
& \geq {\left[I\left(X_{Q} ; U, \hat{X}_{1 Q} \mid Z_{Q}\right)-H\left(\hat{X}_{1 Q} \mid Z_{Q}\right)\right]_{+} } \\
& R_{0}+R_{1}+\epsilon \geq I\left(X_{Q} ; V \mid Y_{Q}\right), \\
& R_{0}+R_{1}+\epsilon \geq[ {\left[\left(X_{Q} ; U, \hat{X}_{1 Q} \mid Z_{Q}\right)-H\left(\hat{X}_{1 Q} \mid Z_{Q}\right)\right]_{+} } \\
&+I\left(X_{Q} ; V \mid U, \hat{X}_{1 Q}, Y_{Q}\right) .
\end{aligned}
$$

Define the following deterministic reconstruction mappings

$$
\begin{aligned}
g_{1}\left(V, Y_{Q}\right) & =\psi_{1 Q}\left(M_{0}, M_{1}, Y^{n}\right), \\
g_{2}\left(U, \hat{X}_{1 Q}, Z_{Q}\right) & =\psi_{2 Q}\left(M_{0}, \hat{X}_{1}^{n}, Z^{n}\right) .
\end{aligned}
$$

Then, the distortion constraints are rewritten as

$$
\begin{aligned}
D_{1}+\epsilon & \geq \mathbb{E}\left[d\left(X^{n}, \psi_{1}\left(M_{0}, M_{1}, Y^{n}\right)\right)\right] \\
& =\mathbb{E}\left[d\left(X_{Q}, g_{1}\left(V, Y_{Q}\right)\right)\right], \\
D_{2}+\epsilon & \geq \mathbb{E}\left[d\left(X^{n}, \psi_{2}\left(M_{0}, \hat{X}_{1}^{n}, Z^{n}\right)\right)\right]
\end{aligned}
$$

$$
=\mathbb{E}\left[d\left(X_{Q}, g_{2}\left(U, \hat{X}_{1 Q}, Z\right)\right)\right] .
$$

We notice that the distribution of $\left(X_{Q}, Y_{Q}, Z_{Q}\right)$ is the same as $(X, Y, Z), \hat{X}_{1 Q}=g_{1}\left(V, Y_{Q}\right)$ and

$$
Z_{Q}-Y_{Q}-X_{Q}-V-U \text {. }
$$

We can therefore replace $\left(X_{Q}, Y_{Q}, Z_{Q}\right)$ by $(X, Y, Z)$ and redefine $\hat{X}_{1}=\hat{X}_{1 Q}$, cf. [11, Section 15.8].

\section{B. Proof of Proposition 1}

The achievability part uses similar arguments to the proof of Theorem 1 with the following changes.

Decoder 2 looks for the unique pair of indices $\left(\tilde{l}_{u}, \tilde{t}_{u}\right)$ such that $\left(u^{n}(\tilde{m}), v^{n}\left(\tilde{m}, l^{\prime}\right), z^{n}, \hat{t}_{1}^{n}\right) \in \mathcal{T}_{\epsilon}^{n}$. In the last step we use the same reasoning as in (22) with $V^{\prime}=\hat{T}_{1}$ as $\hat{T}_{1}=q\left(\hat{X}_{1}\right)$.

The converse is obtained similarly to the proof of Theorem 1. We replace $\hat{X}_{1 i}$ by $\hat{T}_{1 i}$ whenever it appears in rate expressions, since $\hat{T}_{1 i}$ is also a deterministic function of $M_{0}, M_{1}, Y^{n}$. We also keep the definition of $g_{1}(\cdot)$ as in Theorem 1 and observe that $\hat{T}_{1 Q}=q\left(\hat{X}_{1 Q}\right)$. The definition of $g_{2}(\cdot)$ is modified accordingly.

\section{Proof of Theorem 2}

Achievability: Fix a conditional p.m.f $p_{U \hat{X}_{1} \mid X Y}$ and a reconstruction mapping $g_{2}$ such that

$$
\mathbb{E}\left[d\left(X, \hat{X}_{1}\right)\right] \leq D_{1}, \quad \mathbb{E}\left[d\left(X, g_{2}(U, Y)\right)\right] \leq D_{2} .
$$

Our codebook generation, which has the same spirit as in [8, Section II, Remark 6], is similar to [14]. The codebook is deliberately constructed such that given available messages and the current side information symbol $y_{i}$, both decoders are able to identify the output symbol $\hat{x}_{k i}$ uniquely for all $k=1,2$ and $i=1, \ldots, n$, so that the decoding process is causal. We perform encoding in $B$ blocks. For that purpose we need to generate $B$ i.i.d. codebooks. Each of them is described in detail as follows.

Codebook generation: For each $y \in \mathcal{Y}$, we generate $2^{n R_{U}}$ codewords $u^{n}(m \mid y)$ i.i.d. where the $i$-th symbol is drawn using the conditional distribution $p_{U \mid Y}\left(u_{i} \mid y\right)$. After observing a sequence $y^{n}$ the encoder forms a set of $2^{n R_{U}}$ codewords $u^{n}\left(m \mid y^{n}\right)$ where $u_{i} \sim p\left(u_{i} \mid y_{i}\right)$, i.e., the $i$-th symbol of $u^{n}\left(m \mid y^{n}\right)$ is selected as the $i$-th symbol of the $m$-th codeword corresponding to $y_{i}$. Each index $m$ is parsed into a pair $\left(m_{u}, l_{u}\right)$ of the same size. The construction is illustrated in Fig. 4.

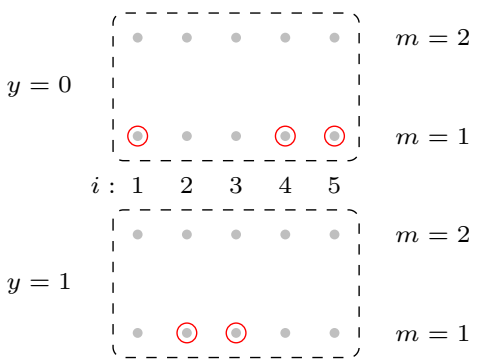

Fig. 4: A visualization of forming sequences $u^{n}\left(m \mid y^{n}\right)$. For the corresponding sequence $y^{n}=(0,1,1,0,0)$ the codeword $u^{n}\left(1 \mid y^{n}\right)$ is marked with red circles. We note that these codewords are conditionally independent given $y^{n}$. 
For each index $m$ and $y \in \mathcal{Y}$, draw $2^{n\left(R_{U}+R_{X_{1}}\right)}$ codewords $\hat{x}_{1}^{n}\left(m, m^{\prime}, l \mid y\right)$, whose $i$-th symbol is generated via $p_{\hat{X}_{1} \mid U Y}\left(\cdot \mid u_{i}(m \mid y), y\right)$. This implies that for each pair of sequences $\left(y^{n}, u^{n}\left(m \mid y^{n}\right)\right)$, the encoder forms a set of $2^{n\left(R_{U}+R_{X_{1}}\right)}$ codewords $\hat{x}_{1}^{n}\left(m, m^{\prime}, l \mid y^{n}\right)$ where the $i$-th symbol of $\hat{x}_{1}^{n}\left(m, m^{\prime}, l \mid y^{n}\right)$ is chosen from the $i$-th symbol of the $\left(m^{\prime}, l\right)$-th codeword corresponding to $\left(y_{i}, u_{i}\left(m \mid y_{i}\right)\right)$. Each index $l$ is parsed into a pair $\left(m_{x_{1}}, l_{x_{1}}\right)$. Later the index $m^{\prime}$ is used for forward encoding. The codebook is revealed to all parties.

Encoding: We use the forward encoding and block Markov decoding approach [7], [15]. Assume that the pair of sequences $\left(x^{n B}, y^{n B}\right)$ are available at the encoder. In the following we use the notation $a^{n}(b)$ to represent the $b$-th block of length $n$ in the sequence $a^{n B}$, where $a$ is understood from the context.

For $b=1$ we search for a codeword $u^{n}\left(m^{1} \mid y^{n}(1)\right)$ in the first codebook, corresponding to the sequence $y^{n}(1)$, such that

$$
\left(x^{n}(1), y^{n}(1), u^{n}\left(m^{1} \mid y^{n}(1)\right)\right) \in \mathcal{T}_{\epsilon}^{n} .
$$

Additionally, for $b \in[1: B-1]$ we also look for a codeword $u^{n}\left(m^{b+1} \mid y^{n}(b+1)\right)$ in the $(b+1)$-th codebook such that

$$
\left(x^{n}(b+1), y^{n}(b+1), u^{n}\left(m^{b+1} \mid y^{n}(b+1)\right)\right) \in \mathcal{T}_{\epsilon}^{n} .
$$

In the next phase, for $b \in[1: B-1]$ the encoder searches for a sequence $\hat{x}_{1}^{n}(\cdot)$ in the $b$-th codebook such that $\left(x^{n}(b), y^{n}(b), u^{n}\left(m^{b} \mid y^{n}(b)\right), \hat{x}_{1}^{n}\left(m^{b}, m^{b+1}, l^{b} \mid y^{n}(b)\right)\right) \quad \in$ $\mathcal{T}_{\epsilon}^{n}$. In the last block we fix $m^{B+1}=(1,1)$ and search for a sequence $\hat{x}_{1}^{n}\left(m^{B},(1,1), l^{B} \mid y^{n}(B)\right)$ in the $B$-th codebook similarly. Note that if there is more than one suitable codewords we select one of them uniformly at random. Otherwise, if no suitable codeword is found, we select a codeword uniformly at random from the corresponding subcodebook.

In the first block, the tuple $\left(m^{1}, m_{u}^{2}, m_{x_{1}}^{1}\right)$ is sent as message $m_{0}$ on the common link while $\left(l_{u}^{2}, l_{x_{1}}^{1}\right)$ is transmitted as $m_{1}$ over the private link. For $b \in[2: B-1]$, the encoder sends the pair $\left(m_{u}^{b+1}, m_{x_{1}}^{b}\right)$ as message $m_{0}$ on the common link and $\left(l_{u}^{b+1}, l_{x_{1}}^{b}\right)$ as message $m_{1}$ on the private link to Decoder 1 . In the last block only $m_{x_{1}}^{B}$ is sent on the common link and $l_{x_{1}}^{B}$ on the private link.

Decoding at Decoder 1: - For $b \in[1: B-1]$, Decoder 1 outputs the sequence $\hat{x}_{1}^{n}\left(m^{b}, m^{b+1}, l^{b} \mid y^{n}(b)\right)$ symbolwisely. We note that, due to our codebook construction, $\hat{x}_{1 i}$ is uniquely determined given $y_{i}(b), m^{b}, m^{b+1}$ and $l^{b}$ for all $i=1, \ldots, n$. Therefore, the decoding process does not depend on the side information non-causally. In the last block, Decoder 1 produces the sequence $\hat{x}_{1}^{n}\left(m^{B},(1,1), l^{B} \mid y^{n}(B)\right)$ in a similar way.

Decoder 2 - In the first block Decoder 2 produces the sequence $\hat{x}_{2}^{n}\left(1 \mid y^{n}(1)\right)$ as $\hat{x}_{2 i}=g_{2}\left(u_{i}\left(m^{1} \mid y_{i}(1)\right), y_{i}(1)\right)$ for all $i=1, \ldots, n$. For $b \in[2: B]$, using the cribbed sequence $\hat{x}_{1}^{n}(b-1)$ and side information $y^{n}(b-1)$ from the previous block Decoder 2 seeks a unique $\hat{l}_{u}^{b}$ such that

$$
\left(u^{n}\left(\hat{m}^{b-1} \mid y^{n}(b-1)\right), \hat{x}_{1}^{n}\left(\hat{m}^{b-1}, \hat{m}^{b}, \tilde{l}^{b-1} \mid y^{n}(b-1)\right),\right.
$$

$$
\left.\hat{x}_{1}^{n}(b-1), y^{n}(b-1)\right) \in \mathcal{T}_{\epsilon}^{n},
$$

for some $\tilde{l}^{b-1}$, where $\hat{m}^{b-1}=\left(m_{u}^{b-1}, \hat{l}_{u}^{b-1}\right), \hat{m}^{b}=\left(m_{u}^{b}, \hat{l}_{u}^{b}\right)$, and $\tilde{l}^{b-1}=\left(m_{x_{1}}^{b-1}, \tilde{l}_{x_{1}}^{b-1}\right)$. Then it outputs the sequence $\hat{x}_{2}^{n}(b)$ as $\hat{x}_{2 i}(b)=g_{2}\left(u_{i}\left(\hat{m}^{b} \mid y_{i}(b)\right), y_{i}(b)\right)$ for all $i=1, \ldots, n$. The decoding process at Decoder 2 has the block Markov manner since it depends on the past block.

Note that given $y_{i}(b)$ and $\hat{m}^{b}$ (or $m^{1}$ ), $u_{i}$ is uniquely determined for all $i=1, \ldots, n$, which implies the decoding process at Decoder 2 is causal.

Analysis: Recall that the distortion measures at both decoders are bounded. Therefore, if $B$ is sufficiently large then all the rates and distortions are close to desired expressions, which are

$$
R_{0}=R_{m_{u}}+R_{m_{x_{1}}}, \quad R_{1}=R_{l_{u}}+R_{l_{x_{1}}} .
$$

From the covering lemma [10], the probability that the chosen codewords and the source pair are jointly typical in each block goes to one as $n \rightarrow \infty$ if

$$
\begin{aligned}
R_{m_{u}}+R_{l_{u}} & >I(X ; U \mid Y)+\gamma_{n}, \\
R_{m_{x_{1}}}+R_{l_{x_{1}}} & >I\left(X ; \hat{X}_{1} \mid U, Y\right)+\gamma_{n} .
\end{aligned}
$$

Assume that Decoder 2 operates correctly in the previous block. It can be shown that when

$$
R_{l_{u}}+R_{l_{x_{1}}}<H\left(\hat{X}_{1} \mid U, Y\right)-\gamma_{n}
$$

Decoder 2 recovers $\hat{m}^{b}=m^{b}$ with probability going to one, which implies that the probability of the event $\left(u^{n}\left(\hat{m}^{b} \mid y^{n}(b)\right), y^{n}(b), x^{n}(b)\right) \in \mathcal{T}_{\epsilon}^{n}$ goes to one as $n \rightarrow \infty$ by our code construction. Therefore, the distortion levels at both decoders are given by

$$
\mathbb{E}\left[d\left(X^{n}, \hat{X}_{k}^{n}\right)\right]<D_{k}+\gamma_{n}, k=1,2 .
$$

Applying the Fourier-Motzkin method the achievable rate region is simplified as

$$
\begin{aligned}
R_{0}+R_{1} & \geq I\left(X ; U, \hat{X}_{1} \mid Y\right), \\
R_{1} & \leq H\left(\hat{X}_{1} \mid U, Y\right),
\end{aligned}
$$

which implies that the region

$$
\begin{aligned}
R_{0} & \geq\left[I\left(X ; U, \hat{X}_{1} \mid Y\right)-H\left(\hat{X}_{1} \mid U, Y\right)\right]_{+} \\
R_{0}+R_{1} & \geq I\left(X ; U, \hat{X}_{1} \mid Y\right),
\end{aligned}
$$

is achievable. This can be seen as each point in (41) dominates a point in (40).

Converse: Assume that there exists a strictly causal code such that the rate-distortion tuple $\left(R_{0}, R_{1}, D_{1}, D_{2}\right)$ is achievable. Denote $M_{k}=\phi_{k}\left(X^{n}, Y^{n}\right)$ for $k=0,1$. Furthermore, define

$$
U_{i}=\left(M_{0}, \hat{X}_{1}^{i-1}, Y^{i-1}\right), \forall i=1, \ldots, n .
$$

Then

$$
\begin{aligned}
n\left(R_{0}\right. & \left.+R_{1}+\epsilon\right) \geq I\left(X^{n}, Y^{n} ; M_{0}, M_{1}\right) \\
& =\sum_{i=1}^{n} I\left(X_{i}, Y_{i} ; M_{0}, M_{1}, X^{i-1}, Y^{i-1}\right)
\end{aligned}
$$




$$
\begin{aligned}
& \stackrel{(a)}{=} \sum_{i=1}^{n} I\left(X_{i}, Y_{i} ; M_{0}, M_{1}, X^{i-1}, Y^{i-1}, \hat{X}_{1}^{i-1}\right) \\
& \geq \sum_{i=1}^{n} I\left(X_{i} ; M_{0}, M_{1}, Y^{i-1}, \hat{X}_{1}^{i-1} \mid Y_{i}\right) \\
& \stackrel{(b)}{=} \sum_{i=1}^{n} I\left(X_{i} ; M_{0}, M_{1}, Y^{i-1}, \hat{X}_{1}^{i-1}, \hat{X}_{1 i} \mid Y_{i}\right) \\
& \geq \sum_{i=1}^{n} I\left(X_{i} ; U_{i}, \hat{X}_{1 i} \mid Y_{i}\right)
\end{aligned}
$$

where

- (a) is because of $\hat{X}_{1 j}=\psi_{1 j}\left(M_{0}, M_{1}, Y^{j}\right)$ for $j=$ $1, \ldots, i-1$,

- (b) holds since $\hat{X}_{1 i}=\psi_{1 i}\left(M_{0}, M_{1}, Y^{i}\right)$.

Similarly,

$$
\begin{aligned}
n\left(R_{0}+\right. & \epsilon) \geq I\left(X^{n}, Y^{n} ; M_{0}\right) \geq I\left(X^{n} ; M_{0} \mid Y^{n}\right) \\
\geq & I\left(X^{n} ; M_{0}, \hat{X}_{1}^{n} \mid Y^{n}\right)-H\left(\hat{X}_{1}^{n} \mid M_{0}, Y^{n}\right) \\
= & \sum_{i=1}^{n} I\left(X_{i} ; M_{0}, \hat{X}_{1}^{n}, Y^{n \backslash i}, X^{i-1} \mid Y_{i}\right) \\
& \quad H\left(\hat{X}_{1 i} \mid M_{0}, Y^{n}, \hat{X}_{1}^{i-1}\right) \\
\geq & \sum_{i=1}^{n} I\left(X_{i} ; U_{i}, \hat{X}_{1 i} \mid Y_{i}\right)-H\left(\hat{X}_{1 i} \mid U_{i}, Y_{i}\right) .
\end{aligned}
$$

Define $U=\left(U_{Q}, Q\right)$ and $g_{2}\left(U, Y_{Q}\right)=\psi_{2 Q}\left(M_{0}, \hat{X}_{1}^{Q-1}, Y^{Q}\right)$, where $Q$ is defined as before. The distortion expressions can be reformulated as

$$
\begin{aligned}
D_{1}+\epsilon & \geq \mathbb{E}\left[d\left(X^{n}, \hat{X}_{1}^{n}\right)\right]=\mathbb{E}\left[d\left(X_{Q}, \hat{X}_{1 Q}\right)\right] \\
D_{2}+\epsilon & \geq \frac{1}{n} \sum_{i=1}^{n} \mathbb{E}\left[d\left(X_{i}, \psi_{2 i}\left(M_{0}, \hat{X}_{1}^{i-1}, Y^{i-1}\right)\right)\right] \\
& =\mathbb{E}\left[d\left(X_{Q}, g_{2}\left(U, Y_{Q}\right)\right)\right],
\end{aligned}
$$

while the rate constraints (43) and (44) become

$$
\begin{aligned}
R_{0}+\epsilon & \geq\left[I\left(X_{Q} ; U, \hat{X}_{1 Q} \mid Y_{Q}\right)-H\left(\hat{X}_{1 Q} \mid U, Y_{Q}\right)\right]_{+} \\
R_{0}+R_{1}+\epsilon & \geq I\left(X_{Q} ; U, \hat{X}_{1 Q} \mid Y_{Q}\right) .
\end{aligned}
$$

As before $\left(X_{Q}, Y_{Q}\right)$ can be replaced by $(X, Y)$ and $\hat{X}_{1 Q}$ is redefined as $\hat{X}_{1}$.

\section{Proof of Proposition 2}

The achievability proof proceeds similarly to the one of Theorem 2 with the following modifications. Using the available causal side information, Decoder 2 outputs the sequence $\hat{x}_{2}^{n}(b)$ as $\hat{x}_{2 i}(b)=g_{2}\left(u_{i}\left(\hat{m}^{b} \mid y_{i}\right), \hat{x}_{1 i}, y_{i}\right)$ for all $i=1, \ldots, n$. Note that if $\hat{m}^{b}$ is correctly recovered then $\left(u^{n}\left(\hat{m}^{b} \mid y^{b}(b)\right), \hat{x}_{1}^{n}(b), y^{n}(b), x^{n}(b)\right) \in \mathcal{T}_{\epsilon}^{n}$. The distortion at Decoder 2 can be bounded by standard arguments.

The converse follows identically as in Theorem 2 .

\section{EXAMPLE}

In this section, we look at the Gerrish source [16] where $\mathcal{X}=\{1,2,3\}$. This distribution of $X$ is given by $p_{X}=$ $\left[\frac{1-p}{2}, p, \frac{1-p}{2}\right]$. We assume that the side information at both decoders is non-causally available, identical and given in [17, Example D] as

$$
Y=\left\{\begin{array}{lll}
1 & \text { if } & X \in\{1,3\} \\
2 & \text { if } & X=2 .
\end{array}\right.
$$

The distortion measure at both decoders is the absolute error, i.e., $d(x, y)=|x-y|$.

The successive refinement region for an identical side information setting is given by [9] as

$$
\begin{aligned}
R_{0} & \geq I(X ; U \mid Y), \\
R_{0}+R_{1} & \geq I(X ; V \mid Y), \\
D_{1} & \geq \mathbb{E}\left[d\left(X, g_{1}(V, Y)\right)\right], \\
D_{2} & \geq \mathbb{E}\left[d\left(X, g_{2}(U, Y)\right)\right],
\end{aligned}
$$

under the constraint $U-V-X-Y$. The cribbing ratedistortion region differs from (48) in

$$
\begin{aligned}
& R_{0} \geq\left[I\left(X ; U, \hat{X}_{1} \mid Y\right)-H\left(\hat{X}_{1} \mid Y\right)\right]_{+}, \\
& D_{2} \geq \mathbb{E}\left[d\left(X, g_{2}^{\prime}\left(U, \hat{X}_{1}, Y\right)\right)\right],
\end{aligned}
$$

i.e., (48a) and (48d) are replaced by (49a) and (49b), respectively. We consider only the case that $D_{1} \leq D_{2}$. In the following we calculate the successive refinement region without cooperation explicitly, which can be regarded as an exposition of [17, Example D]. Given $Y=2, X$ is completely known, hence

$$
\begin{gathered}
I(X ; U \mid Y)=p(Y=1) I(X ; U \mid Y=1) \\
\geq p(Y=1) I\left(X ; \hat{X}_{2} \mid Y=1\right),
\end{gathered}
$$

where $\hat{X}_{2}=g_{2}(U, Y)$. Conditioning on $Y=1, X$ is a binary symmetric source on the alphabet $\{1,3\}$. Due to the convexity of the distortion measure, the alphabet $\hat{\mathcal{X}}_{2}$ can be restricted to $\{1,2,3\}$, by [16, Lemma 4.1.3]. Furthermore, by the proof of [16, Lemma 4.1.4] (see also [17]), $\hat{\mathcal{X}}_{2}$ is $\{1,3\}$. The distortion at Decoder 2 for given $Y=1$ is

$$
\begin{aligned}
\mathbb{E}\left[d\left(X, \hat{X}_{2}\right) \mid Y=1\right]= & 2\left(p\left(X=1, \hat{X}_{2}=3 \mid Y=1\right)\right. \\
& \left.+p\left(X=3, \hat{X}_{2}=1 \mid Y=1\right)\right) .
\end{aligned}
$$

We observe that the distortion level in (51) is two times as much as the one of the Hamming distortion measure, which implies that

$$
\begin{aligned}
& I\left(X ; \hat{X}_{2} \mid Y=1\right) \geq R_{b}\left(\mathbb{E}\left[d\left(X, \hat{X}_{2}\right) \mid Y=1\right] / 2\right) \\
& \geq R_{b}\left(\frac{1}{2 p(Y=1)}\left(D_{2}-p(Y=2) \mathbb{E}\left[d\left(X, \hat{X}_{2}\right) \mid Y=2\right]\right)\right) \\
& \geq R_{b}\left(\frac{D_{2}}{2 p(Y=1)}\right)=R_{b}\left(\frac{D_{2}}{2(1-p)}\right)
\end{aligned}
$$

where $R_{b}(D)=\left(1-h_{b}(D)\right) \mathbf{1}_{\{D \leq 1 / 2\}}$ is the rate-distortion function of the binary symmetric source in which $h_{b}(\cdot)$ is the binary entropy function. Note that $R_{b}(D)$ is a nonincreasing function that is also strictly decreasing on $[0,1 / 2]$. Combining (50) and (52), we obtain

$$
I(X ; U \mid Y) \geq(1-p) R_{b}\left(\frac{D_{2}}{2(1-p)}\right),
$$




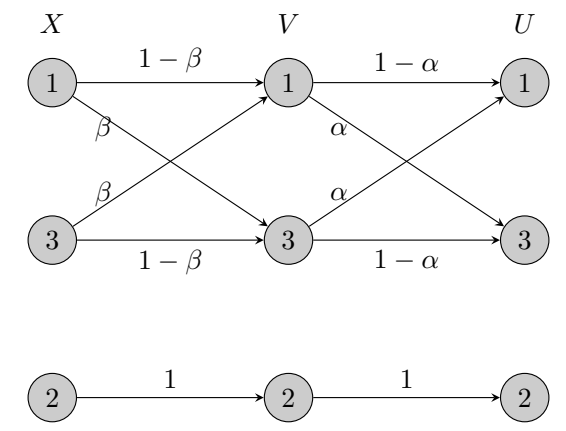

Fig. 5: Illustration of choices of random variables $U, V$. If $D_{1} \leq D_{2}<1-p$ then the parameters $\alpha$ and $\beta$ are $\beta=$ $\frac{D_{1}}{2(1-p)}$ and $\beta \star \alpha=\frac{D_{2}}{2(1-p)}$, where $a \star b=a(1-b)+b(1-a)$. We set $\alpha(\beta)$ to be $1 / 2$ if $D_{2}>1-p$ (or $D_{1}>1-p$ ).

where equality holds when $g_{2}(U, Y)=U$ and $U$ is chosen as in Fig. 5. Arguing similarly, we have

$$
I(X ; V \mid Y) \geq(1-p) R_{b}\left(\frac{D_{1}}{2(1-p)}\right)
$$

where equality holds when $g_{1}(V, Y)=V$ and $V$ is chosen according to Fig. 5. Hence, the achievable rate region for given $D_{1} \leq D_{2}$ of the successive refinement scheme is given by

$$
\begin{aligned}
R_{0} & \geq(1-p) R_{b}\left(\frac{D_{2}}{2(1-p)}\right), \\
R_{0}+R_{1} & \geq(1-p) R_{b}\left(\frac{D_{1}}{2(1-p)}\right) .
\end{aligned}
$$

To obtain an inner bound rate region in the cribbing scheme in the case $D_{1} \leq D_{2}$ we select $\hat{X}_{1}=V$ and $g_{2}^{\prime}\left(U, \hat{X}_{1}, Y\right)=U$, where $U, V$ are given in Fig. 5, then the distortion levels $D_{1} \leq D_{2}$ are guaranteed. The rate constraint on the common link can be omitted, since the right-hand side of (49a) is equal to 0 , while the sum rate constraint (48b) becomes

$$
R_{0}+R_{1} \geq(1-p) R_{b}\left(\frac{D_{1}}{2(1-p)}\right)
$$

We observe that for distortion levels $D_{1} \leq D_{2}$, by dropping the constraint on $R_{0}$ in (49a) and using (54), (56) gives an outer bound of the cribbing rate region. Therefore, (56) provides the achievable rate region for given distortion levels $D_{1} \leq D_{2}$ with the choice of random variables and reconstruction mappings as in the successive refinement scheme without cribbing. Note that, at the boundary of the cribbing region, Decoder 2 also can select $g_{2}^{\prime}\left(U, \hat{X}_{1}, Y\right)=$ $\hat{X}_{1}=V$, which means that its distortion level is $D_{1}$. An example of achievable rate regions in the case $D_{1} \leq D_{2}$ is depicted in Fig. 6.

\section{REFERENCES}

[1] F. Willems, "The discrete memoryless multiple access channel with partially cooperating encoders (corresp.)," IEEE Trans. Inf. Theory, vol. 29 , no. 3 , pp. $441-445,1983$.

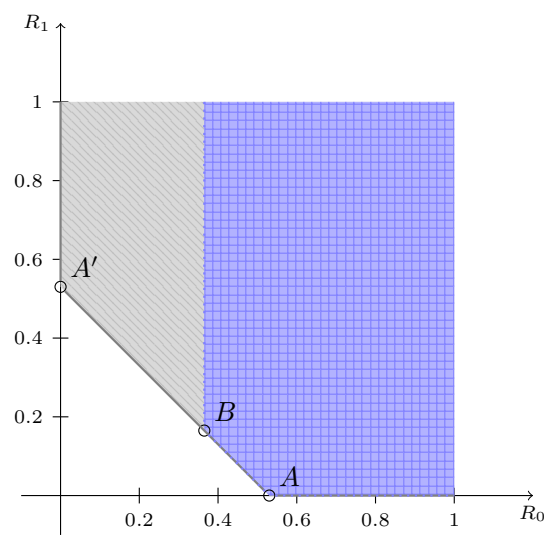

Fig. 6: Achievable rate regions when $D_{1}=0.1$ and $D_{2}=$ 0.2 for $p=0.2$. The successive refinement without cribbing region is filled with blue with extreme points $A$ and $B$. The cribbing one is limited in gray, and is bounded by the line between $A$ and $A^{\prime}$.

[2] F. Willems and E. Van der Meulen, "The discrete memoryless multiple-access channel with cribbing encoders," IEEE Trans. Inf. Theory, vol. 31, no. 3, pp. 313-327, 1985.

[3] A. Bracher and A. Lapidoth, "Feedback, cribbing, and causal state information on the multiple-access channel," IEEE Trans. Inf. Theory, vol. 60 , no. 12, pp. 7627-7654, 2014.

[4] L. Dikstein, H. H. Permuter, and Y. Steinberg, "On state-dependent degraded broadcast channels with cooperation," IEEE Trans. Inf. Theory, vol. 62, no. 5, pp. 2308-2323, 2016.

[5] H. Yamamoto, "Source coding theory for cascade and branching communication systems," IEEE Trans. Inf. Theory, vol. 27, no. 3, pp. 299-308, 1981

[6] Y.-K. Chia, H. H. Permuter, and T. Weissman, "Cascade, triangular, and two-way source coding with degraded side information at the second user," IEEE Trans. Inf. Theory, vol. 58, no. 1, pp. 189-206, 2012.

[7] H. Asnani, H. H. Permuter, and T. Weissman, "Successive refinement with decoder cooperation and its channel coding duals," IEEE Trans. Inf. Theory, vol. 59, no. 9, pp. 5511-5533, 2013.

[8] T. Weissman and A. El Gamal, "Source coding with limited-lookahead side information at the decoder," IEEE Trans. Inf. Theory, vol. 52, no. 12, pp. 5218-5239, 2006.

[9] C. Tian and S. N. Diggavi, "On multistage successive refinement for Wyner-Ziv source coding with degraded side informations," IEEE Trans. Inf. Theory, vol. 53, no. 8, pp. 2946-2960, 2007.

[10] A. El Gamal and Y.-H. Kim, Network information theory. Cambridge university press, 2011.

[11] T. M. Cover and J. A. Thomas, Elements of information theory. John Wiley \& Sons, 2012.

[12] P. Minero, S. H. Lim, and Y.-H. Kim, "A unified approach to hybrid coding," IEEE Trans. Inf. Theory, vol. 61, no. 4, pp. 1509-1523, 2015.

[13] I. B. Gattegno, Z. Goldfeld, and H. H. Permuter, "FME-IT package for MATLAB," http://www.ee.bgu.ac.il/ fmeit/, 2015.

[14] R. Kolte, A. Ozgur, and H. Permuter, "Cooperative binning for semideterministic channels," IEEE Trans. Inf. Theory, vol. 62, no. 3, pp. 1231-1249, 2016.

[15] P. Cuff and L. Zhao, "Coordination using implicit communication," in Information Theory Workshop. IEEE, 2011, pp. 467-471.

[16] W. H. R. Equitz, "Successive refinement of information," Ph.D. dissertation, Stanford University, 1989.

[17] Y. Steinberg and N. Merhav, "On successive refinement for the WynerZiv problem," IEEE Trans. Inf. Theory, vol. 50, no. 8, pp. 1636-1654, 2004. 